\title{
Strongly nonlinear waves in a chain of Teflon beads
}

\author{
C. Daraio, ${ }^{1}$ V. F. Nesterenko, ${ }^{1,2, *}$ E. B. Herbold, ${ }^{2}$ and S. Jin ${ }^{1,2}$ \\ ${ }^{1}$ Materials Science and Engineering Program, University of California at San Diego, La Jolla, California 92093-0411, USA \\ ${ }^{2}$ Department of Mechanical and Aerospace Engineering, University of California at San Diego, La Jolla, California 92093-0411, USA
}

(Received 11 March 2005; published 6 July 2005)

\begin{abstract}
One-dimensional "sonic vacuum" type phononic crystals were assembled from a chain of polytetrafluoroethylene (PTFE,Teflon) spheres with different diameters in a Teflon holder. It was demonstrated that this polymer-based sonic vacuum, with exceptionally low elastic modulus of particles, supports propagation of strongly nonlinear solitary waves with a very low speed. These solitary waves can be described using the classical nonlinear Hertz law despite the viscoelastic nature of the polymer and high strain rate deformation of the contact area. The experimentally measured speeds of solitary waves at high amplitudes are close to the theoretically estimated values with a Young's modulus of $1.46 \mathrm{GPa}$ obtained from shock wave experiments. This is significantly higher than the Young's modulus of PTFE from ultrasonic measurements. Trains of strongly nonlinear solitary waves excited by an impact were investigated experimentally and were found to be in reasonable agreement with numerical calculations based on Hertz interaction law though exhibiting a significant dissipation.
\end{abstract}

DOI: 10.1103/PhysRevE.72.016603

\section{INTRODUCTION}

The study of strongly nonlinear wave propagation in onedimensional chains of spherical beads representing the simplest model of granular materials has received much attention in recent years [1-32]. One of the distinguishing properties of these materials is the existence of a qualitatively different solitary wave with a finite width that is independent of the solitary wave amplitude. This solitary wave was discovered, in 1983 analytically and numerically [1] and later in 1985 it was observed in experiments [2]. Strongly nonlinear wave dynamics is a natural extension of the weakly nonlinear wave dynamics described by the Korteweg-de Vries $(\mathrm{KdV})$ equation $[4,18]$. Initially strongly precompressed, strongly nonlinear granular chains may behave as weakly nonlinear systems similar to the one considered in the Fermi-Pasta-Ulam paper [33]. In contrast to weakly nonlinear systems, the behavior of strongly nonlinear uncompressed granular chains exhibits qualitatively different features $[4,17,18,23,27,30-32]$. Further applications might arise from understanding the basic physics of these onedimensional (1D) systems. Sound focusing devices (tunable acoustic lenses and delay lines), sound absorption layers, and sound scramblers are among the most promising engineering applications.

Nonclassical, strongly nonlinear wave behavior appears if the granular material is weakly compressed $[1,2,18]$. In this case, the amplitude in a wave is significantly higher than the forces caused by initial precompression. The anharmonic approximation based on the small parameter (ratio of wave amplitude to initial precompression) is not valid. The principal difference between the strongly nonlinear case and the strongly compressed weakly nonlinear chain is due to the lack of a small parameter with respect to the wave amplitude in the former case. The long wave equation for particle displacement $u$ in this case is $[1,18]$

\footnotetext{
*Corresponding author. Email address: vnesterenko@ucsd.edu
}

PACS number(s): 05.45.Yv, 46.40.Cd, 43.25.+y, 45.70.-n

$$
u_{t t}=-c^{2}\left(\left(-u_{x}\right)^{3 / 2}+\frac{a^{2}}{10}\left\{\left(-u_{x}\right)^{1 / 4}\left[\left(-u_{x}\right)^{5 / 4}\right]_{x x}\right\}\right),
$$

where

$$
-u_{x}>0, \quad c^{2}=\frac{2 E}{\pi \rho\left(1-\nu^{2}\right)}, \quad c_{0}=\left(\frac{3}{2}\right)^{1 / 2} c \xi_{0}^{1 / 4} .
$$

Here $E, \rho$, and $\nu$ are the bulk elastic modulus, density, and Poisson ratio of the particles in the chain. The particle diameter is $a$ and $\xi_{0}$ is the initial strain in the system (phononic crystal). It should be mentioned that the constant $c$ is of the same order of magnitude as the bulk sound speed in the particle material and not the sound speed in the phononic crystal. Instead the parameter $c_{0}$ corresponds to a long wave sound speed related to initial strain $\xi_{0}$. This equation for high amplitude pulses (or for negligible precompression) has no characteristic wave speed that is independent of amplitude. The regularized equation and the equation for a general interaction law can be found in [18]. Despite its complex nature Eq. (1) has simple stationary solutions with unique properties that are similar to the stationary solutions for the discrete chain even though some differences due to the relatively short width of solitary wave exist [1,16-18,23,24,29]. The solitary wave with a speed $V_{s}$ in a "sonic vacuum" can be closely approximated by one hump of a periodic solution with finite length $(L)$ equal to only five particle diameters $[1,18]$ :

$$
\begin{aligned}
\xi & =\left(\frac{5 V_{s}^{2}}{4 c^{2}}\right) \cos ^{4}\left(\frac{\sqrt{10}}{5 a} x\right) \\
& =\frac{1}{8}\left(\frac{5 V_{s}^{2}}{4 c^{2}}\right)^{2}\left[3+\cos 4\left(\frac{\sqrt{10}}{5 a} x\right)+4 \cos 2\left(\frac{\sqrt{10}}{5 a} x\right)\right] .
\end{aligned}
$$

The concept of the sonic vacuum was introduced in $[3,5,6,9]$ to emphasize the fact that in an uncompressed chain $\left(\xi_{0}\right.$ $=0)$ the sound speed is equal to zero. The solitary wave speed $V_{s}$ has a nonlinear dependence on maximum strain $\xi_{m}$, 
particle velocity $v_{m}$, and the force between particles $F_{m}$ :

$$
V_{s}=\frac{2}{\sqrt{5}} c \xi_{m}^{1 / 4}=\left(\frac{16}{25}\right)^{1 / 5} c^{4 / 5} v_{m}^{1 / 5}=0.68\left(\frac{2 E}{a \rho^{3 / 2}\left(1-\nu^{2}\right)}\right)^{1 / 3} F_{m}^{1 / 6}
$$

In a weakly compressed chain a supersonic solitary wave $\left(V_{s}>\mathrm{c}_{0}\right)$ with an amplitude much higher than the initial precompression propagates with a speed $V_{s}$, which can also be closely approximated by one hump of the periodic solution corresponding to zero prestress $[1,18]$.

The speed of a wave in a sonic vacuum can be infinitesimally small if the amplitude of the wave is also small. It is interesting that a strongly nonlinear system supports solitary waves that are composed from a constant strain and only two harmonics (with wavelengths about $2.5 a$ and $5 a$, respectively) [see Eq. (2)]. The existence of this unique wave was verified analytically, numerically, and in experiments $[1,2,4,7,8,10,11,13,14,16-18,24]$. This solitary wave can be considered as a soliton in a physically reasonable approximation $[1,4,18]$, though small amplitude secondary solitary waves were observed in numerical calculations after collision of two identical solitary waves. The ratio of the largest amplitude of the secondary wave to the amplitude of the original wave is about 0.02 [21]. This solitary wave is of a fundamental interest because Eq. (1) is more general than the weakly nonlinear $\mathrm{KdV}$ equation, which describes the behavior of various physical systems [12] and the former includes the latter.

The solitary wave speed $V_{s}$ in a chain with finite prestress $\xi_{0}$ due to applied static precompression $F_{0}$ for tuning can be written in terms of normalized maximum strain $\xi_{r}=\xi_{m} / \xi_{0}$ or force $f_{r}=F_{m} / F_{0}[18]$ :

$$
\begin{aligned}
V_{s}= & c_{0} \frac{1}{\left(\xi_{r}-1\right)}\left(\frac{4}{15}\left(3+2 \xi_{r}^{5 / 2}-5 \xi_{r}\right)\right)^{1 / 2} \\
= & 0.9314\left(\frac{4 E^{2} F_{0}}{a^{2} \rho^{3}\left(1-\nu^{2}\right)^{2}}\right)^{1 / 6} \frac{1}{\left(f_{r}^{2 / 3}-1\right)} \\
& \times\left(\frac{4}{15}\left(3+2 f_{r}^{5 / 3}-5 f_{r}^{2 / 3}\right)\right)^{1 / 2} .
\end{aligned}
$$

For simplicity only the leading approximation was used to connect the strains in the continuum limit and the forces in a discrete chain in Eqs. (3) and (4).

It is important to mention that $V_{s}$ can be significantly smaller than the bulk sound speed in the material composing the beads and can be considered approximately constant in any narrow interval of its relative amplitude $f_{r}$. The described properties of strongly nonlinear waves might allow the use of sonic-vacuum-based materials as effective delay lines with exceptionally low speed of signal propagation. The estimation based on Eq. (3) with Young's modulus $E$ $=600 \mathrm{MPa}$, Poisson's ratio $\nu=0.46$, and density $\rho=2.2$ $\times 10^{3} \mathrm{~kg} / \mathrm{m}^{3}$ [34] of polytetrafluoroethylene (PTFE) shows that it is possible to create materials with an impulse speed below $100 \mathrm{~m} / \mathrm{s}$, which corresponds to a particle velocity of $0.2 \mathrm{~m} / \mathrm{s}$ or smaller. This signal speed in condensed soft matter is below the level of sound speed in gases at normal
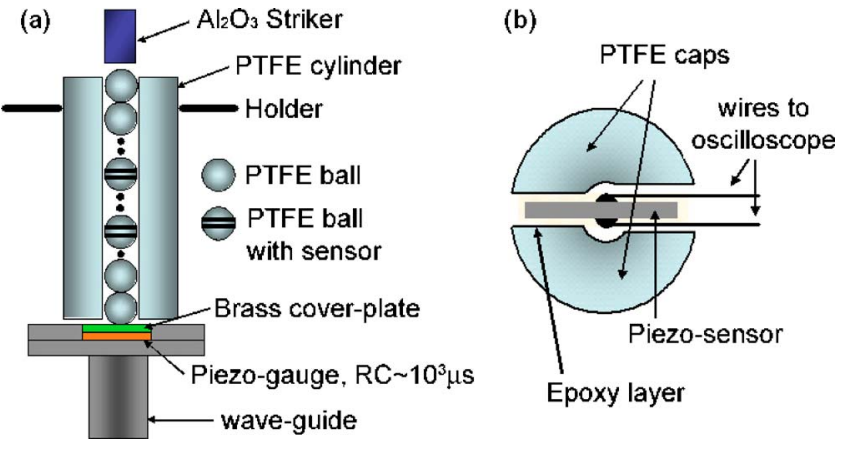

FIG. 1. (Color online) (a) Experimental setup for testing of 1D strongly nonlinear phononic crystals with PTFE beads; (b) schematic drawing of a particle with embedded piezosensor.

conditions. In this paper we present experimental results on pulse propagation in PTFE chains of spheres in accord with the main conclusions of the outlined strongly nonlinear theory. The speed of the signals is in the range of 88 to $168 \mathrm{~m} / \mathrm{s}$. Uniformly compressed discrete chains have been considered in numerical analysis and in experiments $[4,18,25]$. It was shown that the solitary wave speed generated by the impact of a piston with the same velocity increases with precompression. Also, the tendency of the impulse to split into a train of solitary waves decreases and the solitary wave width increases. Gravitationally loaded discrete chains are considered in numerical calculations in papers $[15,19,22]$.

It should be noticed that particles with Hertzian contacts serving as strongly nonlinear springs are not the only way of discovering a sonic vacuum type system. Any power law interaction between particles $(n>1)$ results in a similar behavior $[3,17,18]$. Also, any general strongly nonlinear interaction laws support solitary waves with finite length for the long wave approximation [18]. Different physical systems can be designed with properties suitable for the realization of sonic vacuum type behavior. For example, a forest of vertically aligned carbon nanotubes exhibits strongly nonlinear but non- Hertzian type force interaction with spherical particles which can be used for assembling strongly nonlinear phononic crystals [35].

The solitary wave width for general strongly nonlinear interaction law is proportional to the bead diameter (or distance between particles) and the speed has a nonlinear dependence on amplitude [18]. It is interesting that a power law interaction with $n=3$, corresponding to a physical system of particles on an unstretched string in transverse vibrations $[5,18]$ supports periodic harmonic waves and solitary waves with a linear dependence of maximum strain on speed.

\section{EXPERIMENTAL PROCEDURES AND RESULTS}

One-dimensional phononic crystals were assembled by filling a PTFE tube (with inner diameter $5 \mathrm{~mm}$ ) with chains of 11 and 21 PTFE balls (McMaster-Carr) with diameter $a$ $=4.76 \mathrm{~mm}$ and mass $0.1226 \mathrm{~g}$ (standard deviation $0.0008 \mathrm{~g}$ ) (Fig. 1). Different numbers of particles were used to clarify the stages of impulse transformation and interaction with the 
wall. A chain assembled from 18 PTFE particles with smaller diameter $a=2.38 \mathrm{~mm}$ and mass equal to $0.0157 \mathrm{~g}$ (standard deviation $0.0003 \mathrm{~g}$ ) in a PTFE tube with inner diameter of $2.5 \mathrm{~mm}$ was also tested. Using two different sizes of beads helps to understand the behavior of the investigated polymeric material in the contact area at different stresses, strains, and strain rates, which are dependent on the particle size. Scaling down the particle sizes is important for future applications in different devices (i.e., biomedical application, imaging, sound scrambling, etc.). Waves of different amplitude and duration were excited by impacting the top of the chain with strikers of different mass and velocity.

The experimental setup for measurements of soliton speed, duration, and force amplitude together with the measurements of reflected pulse from the wall is presented in Fig. 1. It includes three calibrated piezosensors $(R C$ $\sim 10^{3} \mu \mathrm{s}$ ) connected to a Tektronix oscilloscope. Two lead zirconate titanate based piezogauges $(3 \mathrm{~mm}$ side plates with thickness $0.5 \mathrm{~mm}$ ) with nickel plated electrodes and custom microminiature wiring, supplied by Piezo Systems, Inc., were embedded inside two PTFE particles as in [18,36,37]. The particles with embedded sensors consisted of two PTFE caps with a total mass $2 M=0.093 \mathrm{~g}$ and a sensor with mass $m=0.023 \mathrm{~g}$ glued between these caps. Including glue, the total mass of the sensor was equal to $0.116 \mathrm{~g}$ [Fig. 1(b)], which was very close to the mass of the PTFE particle, $0.123 \mathrm{~g}$. This design allows a calculation of the speed of solitary wave simultaneously with measurement of the forces acting inside the particles.

A third piezogauge, supplied by Kinetic Ceramics, Inc., was bonded with epoxy on electrode foils for contacts and reinforced by a $1 \mathrm{~mm}$ brass plate on the top surface. The sensor assembly was then placed on the top surface of a long vertical steel rod (waveguide) embedded at the other end into a steel block to avoid possible wave reverberation in the system [Fig. 1(a)]. This sensor was calibrated by using the impact of a single steel ball, which provides similar conditions of loading as in our measurements. The initial velocity and linear momentum conservation law were used for calibration. The area under the force-time curve measured by the gauge was integrated from the beginning of impact up to the point of maximum force and compared with the linear momentum of the particle at the beginning of impact. The sensors in the two particles were calibrated by comparison with the signal from the sensor at the wall. This was done using a controlled, relatively long, simultaneous loading of the particle with the sensor and the sensor in the wall by the impact of a massive piston.

The introduction of a particle with a different mass (particle with a sensor) in the chain of particle of equal masses results in wave reflections investigated in $[11,17,19]$. It was suggested to use reflected signals for detection of buried inclusions [19]. In numerical calculations, a slightly lighter particle with mass $0.116 \mathrm{~g}$ was introduced into the chain of particles with mass $0.123 \mathrm{~g}$, producing wave reflections that would be too small to detect experimentally. Attenuating a solitonlike pulse in a chain of random particles was considered in $[1,4,18,20]$.

To interpret the signal measured in the experiments we considered the particle with an embedded sensor as a rigid (a)
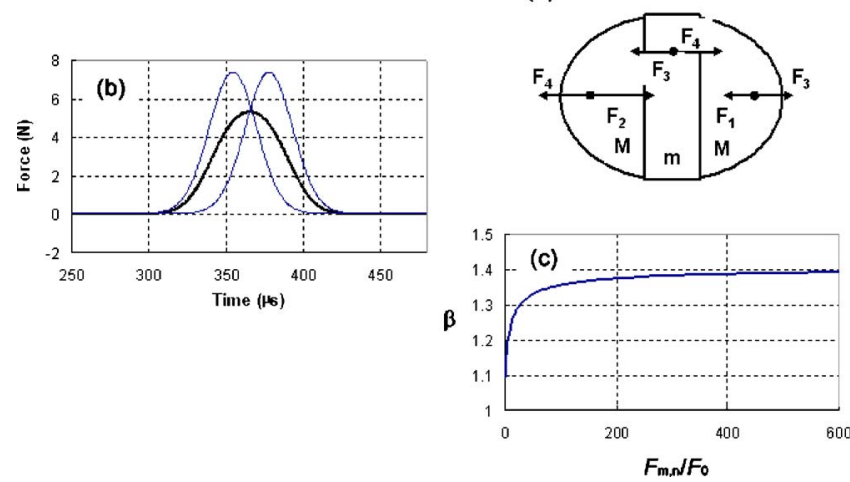

FIG. 2. (Color online) (a) Schematic drawing showing the forces acting on the different parts of particle with embedded sensor. (b) Force vs time plot obtained in numerical calculations for the two contacts of the particle with embedded sensor (left and right curves corresponding to $F_{1}$ and $F_{2}$ ) and the average of the previous two (central curve). (c) Dependence of the coefficient $\beta$ on the amplitude of the normalized dynamic average force $\left(F_{m, n} / F_{0}\right)$ in the solitary wave $\left[F_{m, n}\right.$ is the maximum of the central curve in (b)], found in numerical calculations.

body [Fig. 2(a)]. The forces on the sides of the contacts of the particle $\left(F_{1}\right.$ and $\left.F_{2}\right)$ can be easily related to the forces acting on both sides of the sensor $\left(F_{3}\right.$ and $\left.F_{4}\right)$ :

$$
\begin{aligned}
& F_{3}=\frac{F_{1}+F_{2}}{2}+\frac{F_{1}-F_{2}}{2} \frac{m}{2 M+m}, \\
& F_{4}=\frac{F_{1}+F_{2}}{2}-\frac{F_{1}-F_{2}}{2} \frac{m}{2 M+m} .
\end{aligned}
$$

From Eq. (5) we can see that the average of the compression forces $F_{3}$ and $F_{4}$ (considered positive) is equal to the average value of forces $F_{1}$ and $F_{2}, F \equiv\left(F_{1}+F_{2}\right) / 2$, acting on the particle contacts. It should be mentioned that in numerical modeling the particles are considered rigid bodies and only the contact forces $F_{1}$ and $F_{2}$ are taken into consideration. The time dependence of forces on the particle contacts was calculated numerically and their average values are presented in Fig. 2(b).

In the case of $m \ll M$, the forces on each side of the sensor [Eq. (5)] are very close to the average forces on the particle contacts $[18,36]$. In our case the forces $F_{3}$ and $F_{4}$ deviate from their average value by less than $20 \%$ in the vicinity of signal "shoulders," and are seen from the time dependence of contact forces in Fig. 2(b). It should be noted that at the moment when the averaged force $F$ is maximum it is equal to the corresponding forces $F_{3}$ and $F_{4}$ [Fig. 2(b), Eq. (5)]. Comparison of the averaged force and contact forces [Fig. 2(b)] reveals that averaging reduces the maximum amplitude of the force and increases the duration of the pulse. This averaged curve is used for comparison with experimental results based on sensors embedded in the particles.

To relate the maximum value of average compression force $F_{m, e}$ measured by the embedded sensor to the value of maximum force at the contact between neighboring particles [Fig. 2(a)], we used a coefficient $\beta$ determined in numerical 

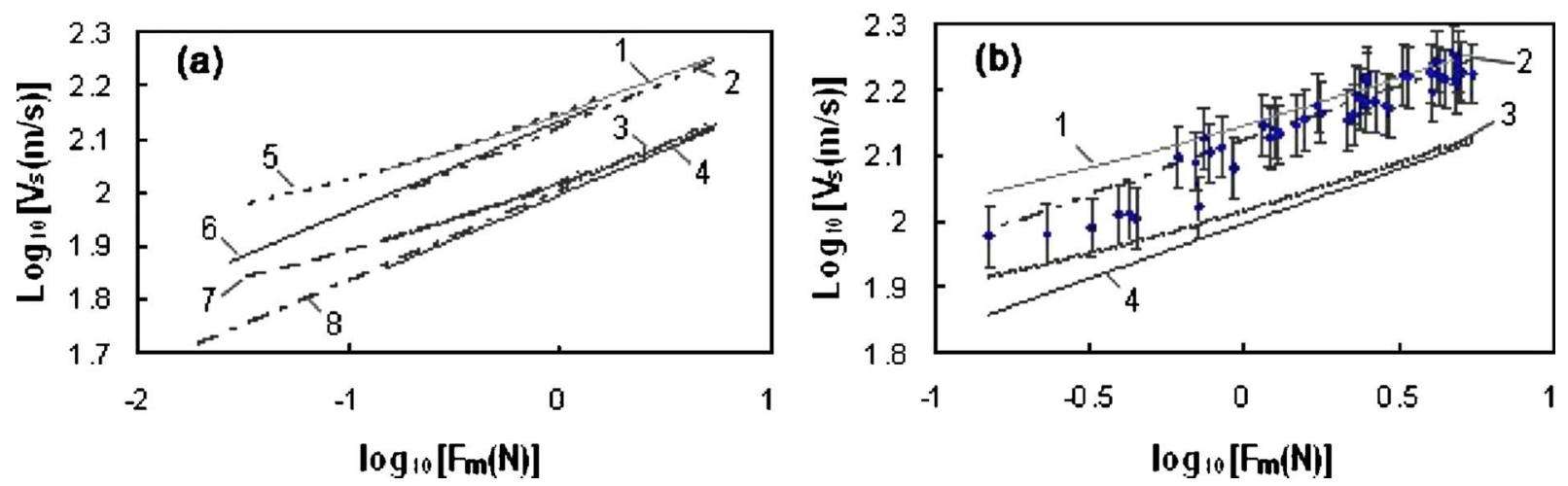

FIG. 3. Dependence of the solitary wave speed on its amplitude. (a) Comparison of numerical results for a discrete chain and analytical data obtained from the long wave approximation. Curves 1 and 3 represent the long wave approximation for gravitationally precompressed systems [Eq. (4)] at $E=1460$ and $600 \mathrm{MPa}$ respectively; curves 5 and 7 represent the corresponding numerical calculations for a discrete chain. Curves 2 and 4 are the theoretical curves based on Eq. (3) for a sonic vacuum with a Young's modulus equal to 1460 and 600 MPa, respectively. Curves 6 and 8 represent the corresponding numerical calculations for these cases. (b) Comparison of the experimental values (shown by solid dots) with the curves obtained from the long wave approximation. Curves 1-4 in (b) are the same as in (a).

calculations. It represents the ratio of the amplitude of the dynamic force on the particle contacts to the maximum average of dynamic forces $F_{m, n}$ acting on the two contacts of the given particle in the solitary wave. Relating the two forces facilitates the use of Eqs. (3) and (4) with experimental data. The dependence of $\beta$ on the normalized force amplitude of a solitary wave is presented in Fig. 2(c). This coefficient has negligible dependence on the elastic moduli of the PTFE particles $(<1 \%$ in the investigated range of solitary wave amplitude and relevant range of elastic modulus from 600 to $1460 \mathrm{MPa}$ ). It should be mentioned that the investigated range of the relative amplitudes of the dynamic force and static precompression represents a strongly nonlinear regime of system behavior resulting in relatively short length solitary waves. The coefficient $\beta$ is about 1 in the linear regime when the amplitude of the dynamic force is much smaller than the initial precompression and the solitary waves are very long in comparison with a particle diameter.

The maximum compression force on the contact between two particles $\left(F_{m}\right)$ adjacent to the particle with the sensor was calculated using the equation:

$$
F_{m}=\beta F_{m, e}+F_{0}
$$

where $F_{m, e}$ is the maximum averaged dynamic compression force measured experimentally by the gauge embedded in the particle (it is represented by $F_{m, n}$ in numerical calculations) and $F_{0}$ is the gravitational precompression.

Pulses of different durations and amplitudes in the 1D phononic crystals were generated by impact of an alumina $\left(\mathrm{Al}_{2} \mathrm{O}_{3}\right)$ cylinder $(0.47 \mathrm{~g})$, a PTFE ball with a diameter of $4.76 \mathrm{~mm}$ (mass $0.123 \mathrm{~g}$ ), or a stainless steel bead with a diameter of $2 \mathrm{~mm}$ (mass $0.036 \mathrm{~g}$ ) onto the top particle of the chain. Single solitary waves can be generated by an impactor with a mass equal to the mass of the beads in the system, which is physically equivalent to the application of a $\delta$-function force $[1,4,18]$. To generate a single solitary wave in a chain of 21 PTFE beads, we used the same bead as the striker $(m=0.123 \mathrm{~g})$. Sensors were placed in the ninth and fifth balls from the bottom and in the wall at the end of the chain.

The theoretically predicted speed of solitary waves in strongly nonlinear phononic crystals has a strong dependence on the amplitude represented by Eqs. (3) and (4) for sonic vacuums and for precompressed chains, respectively. This is shown in Fig. 3(a) together with the corresponding numerical calculations of the soliton speed for discrete chains. The curves based on the long wave approximation [Eqs. (3) and (4)] and the numerically calculated values practically coincide. In experiments [solid dots in Fig. 3(b)], the solitary wave speeds for different amplitudes were obtained by dividing the distance between the sensors by the measured peakto-peak time interval. The corresponding force amplitude in the solitary wave was found based on the measurements of gauges embedded inside the particles. The $\log _{10} F_{m}-\log _{10} V_{s}$ curves presented in Fig. 3(b) are based on these measurements. Accuracy of the measurements of amplitude of solitary waves was in the range of $15 \%$ to $30 \%$ for large and small amplitudes, the larger errors being due to the higher signal to noise ratio at low amplitudes. In experiments the accuracy of the speed measurement can be estimated within $10 \%$ due to the uncertainty in the sensor alignment (about $1 \mathrm{~mm}$ for each sensor).

After measuring the speed and duration of the propagating pulse, the widths of solitary waves were calculated for the corresponding force amplitudes (Table I). The same data obtained from numerical analysis of discrete chains with PTFE elastic modulus $1.46 \mathrm{GPa}$ based on the averaged forces on the particle contacts are also shown with solitary width truncated at the levels $0.2 \%$ and $4 \%$ of the solitary wave amplitude.

Experimental results for forces measured by sensors embedded into the particles and into the wall corresponding to a $2.0 \mathrm{~m} / \mathrm{s}$ impact velocity are shown in Fig. 4(a). The zero time in all experiments corresponds to the start of recording triggered by the signal. In numerical calculations presented in all figures the zero time corresponds to the moment of impact.

One of the distinguished features of a strongly nonlinear sonic vacuum type system is the fast decomposition of shock 
TABLE I. Experimental data for amplitude, speed, duration, and normalized width of solitary wave in the PTFE chain composed of particles with diameter $2 R=4.76 \mathrm{~mm}$. Numerical data for discrete chains are also presented for comparison.

\begin{tabular}{ccccccc}
\hline \hline \multicolumn{5}{c}{ Experimental data } & \multicolumn{3}{c}{ Numerical results } \\
$F_{\mathrm{m}}(\mathrm{N})$ & Duration $(\mu \mathrm{s})$ & $V_{s}(\mathrm{~m} / \mathrm{s})$ & $L / 2 R$ & $V_{s}(\mathrm{~m} / \mathrm{s})$ & $L / 2 R($ at $0.2 \%)$ & $L / 2 R($ at $4 \%)$ \\
\hline 5 & 153 & 168 & 5.4 & 190 & 5.4 & 4.0 \\
2 & 164 & 152 & 5.2 & 164 & 5.7 & 4.1 \\
0.6 & 233 & 106 & 5.2 & 137 & 6.2 & 4.3 \\
0.1 & 326 & 97 & 6.6 & 109 & 7.8 & 5.3 \\
0.06 & 360 & 88 & 6.7 & 103 & 8.6 & 5.7 \\
\hline \hline
\end{tabular}

type pulse caused by impact at a short distance from the impacted side $[1,4,18]$. To check if the PTFE-based strongly nonlinear phononic crystal exhibits this property, impact by a striker with a larger mass $\left(\mathrm{Al}_{2} \mathrm{O}_{3}\right.$ cylinder $\left.0.47 \mathrm{~g}\right)$ was employed to create a longer initial shock pulse in chains of different lengths. This impact results in an incoming pulse shape at the entrance of the system with the rise time equal-
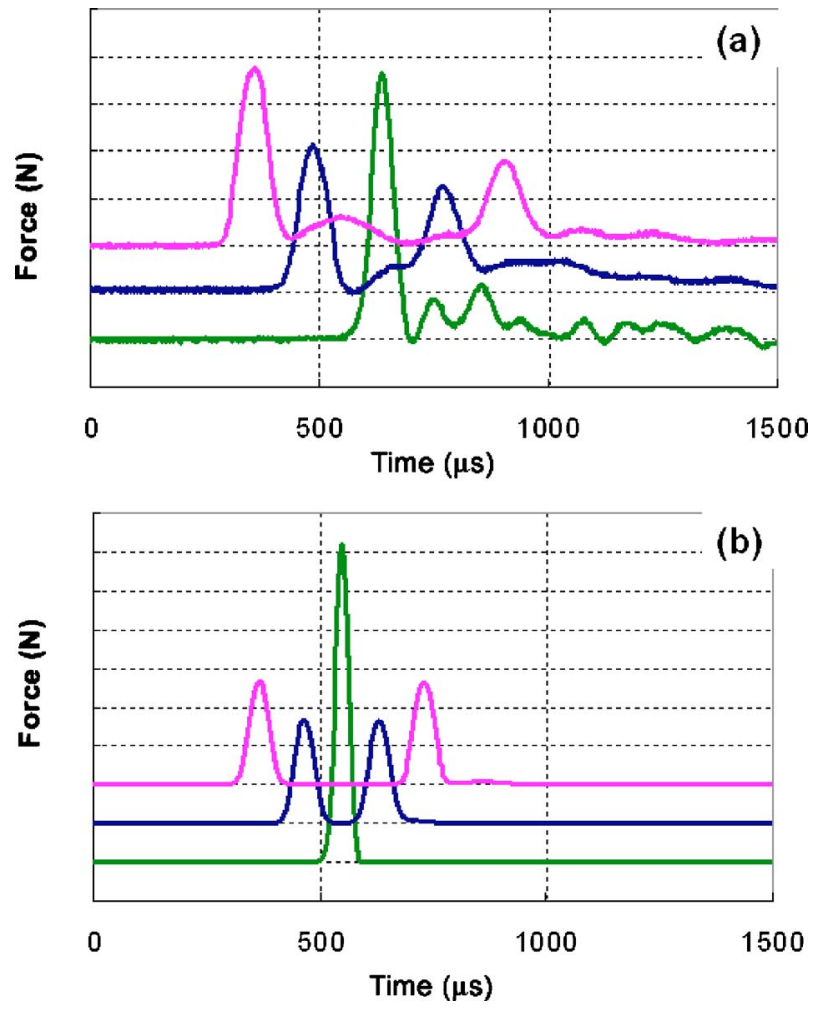

FIG. 4. (Color online) Solitary waves in PTFE chain generated by PTFE ball striker with a velocity of $2 \mathrm{~m} / \mathrm{s}$. (a) Incident and reflected solitary waves, and force on the wall detected experimentally in the chain of 21 PTFE beads with diameter $4.76 \mathrm{~mm}$. The curves represent force vs time detected by the sensor embedded into the ninth ball from the wall (top curve), by the sensor in the fifth ball from the wall (middle curve), and at the wall (the vertical scale is equal to $0.5 \mathrm{~N}$ ). (b) Numerical calculations for a discrete chain under conditions corresponding to experimental conditions in (a). Curves represent the average value of the forces acting on the top and bottom contact of each sensor. Grid scaling on the vertical axes is $2 \mathrm{~N}$. ing $50 \mu \mathrm{s}$ and an initial decay with the characteristic exponential behavior $A \exp (-0.0185 t)$, where the time $(t)$ is measured in microseconds, starting from the peak of the signal with amplitude $A$. The total duration of the incident signal is equal to $370 \mu \mathrm{s}$. The result for the short chain composed of 11 PTFE particles is presented in Fig. 5(a).

It is important to note that if exponential curves are drawn through the soliton maxima, corresponding to the force history at a given point (in Fig. 5 this point corresponds to the wall), they will depend on the position of the sensor due to the dependence of soliton speeds on amplitude. The exponential decays corresponding to the experimental data and numerical calculations at the wall are $A_{e} \exp (-0.0067 t)$ and $A_{n}(\exp -0.0059 t)$ (time is measured in microseconds). The absolute values of coefficients in these exponents are about three times smaller than in the incident pulse (0.0185) due to the dependence of soliton speeds on amplitude. Despite the evident attenuation in experiments $\left(A_{e}<A_{n}\right)$ the corresponding exponents for envelope curves in experimental data and numerical calculations are close to each other. This suggests that the attenuation of the solitary waves is not strongly dependent on their amplitudes at the investigated range of amplitudes.

A chain of smaller diameter PTFE particles $(2.38 \mathrm{~mm})$ was also investigated to determine the diameter dependence of the strongly nonlinear behavior of the PTFE-sphere-based sonic vacuum and its dissipative properties. It should be mentioned that based on the Hertz law the radius of the contact area decreases with decreasing particle radius under the same force. The experimental results are presented in Fig. 6(a) for short duration of shock loading (impact by a $2 \mathrm{~mm}$ diameter steel ball with a mass about 2.3 times that of the PTFE particle) and for relatively long duration of impact induced by a PTFE ball with a diameter $4.76 \mathrm{~mm}$ and mass $0.123 \mathrm{~g}$ [Fig. 6(c)].

\section{NUMERICAL CALCULATIONS}

It was shown previously $[1,2,4,10,14,18]$ that wave propagation in a 1D system of linear elastic beads can be described considering particles as rigid bodies connected by nonlinear springs according to the Hertz law [Eq. (7)] for contact interaction of spheres $[38,39]$ : 

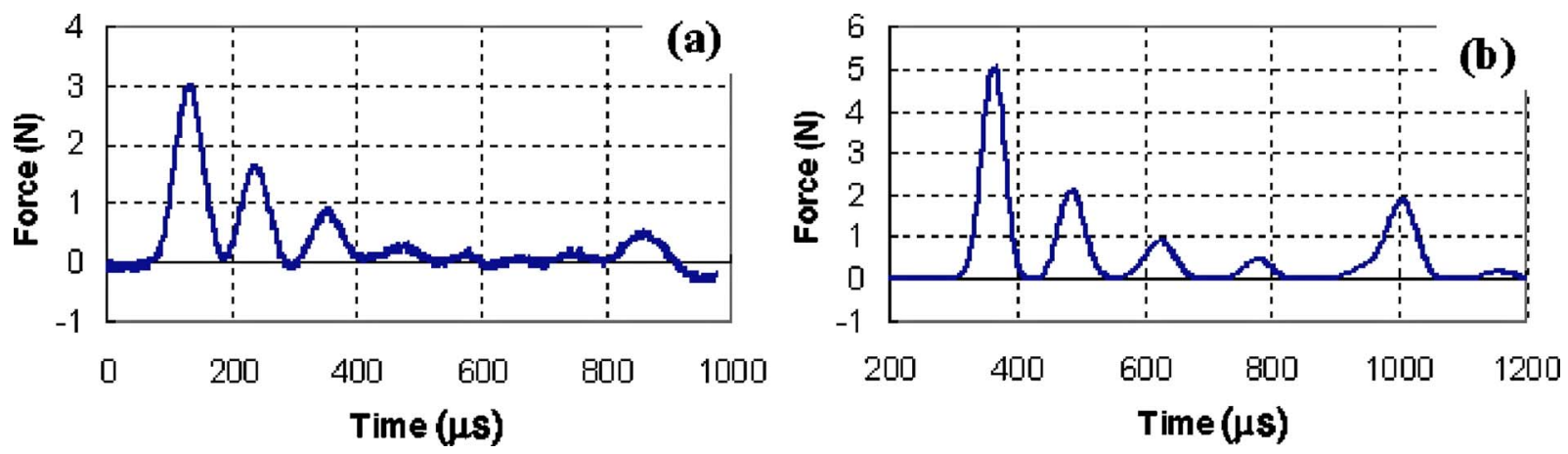

FIG. 5. (Color online) Experimental and theoretical results demonstrating that a short chain of PTFE beads with a diameter 4.76 mm supports a train of solitary waves induced by an alumina striker with a mass equal to four times the mass of the particle. (a) Force detected in experiment by the sensor mounted at the wall supporting an 11-PTFE-particle chain, striker velocity $0.44 \mathrm{~m} / \mathrm{s}$, vertical scale $1 \mathrm{~N}$. (b) Numerical calculations corresponding to experimental conditions in (a), including gravitational precompression; vertical scale is $1 \mathrm{~N}$, elastic is modulus $1.46 \mathrm{GPa}$.

$$
F=\frac{2 E}{3\left(1-\nu^{2}\right)}\left(\frac{R_{1} R_{2}}{R_{1}+R_{2}}\right)^{1 / 2}\left[\left(R_{1}+R_{2}\right)-\left(x_{1}-x_{2}\right)\right]^{3 / 2} .
$$

A system of second order differential equations was reduced to first order equations $[1,4,18]$ and numerical calculations were performed using MATLAB.

For comparison with experiments we calculated an average compression force $F=\left(F_{1}+F_{2}\right) / 2$ for solitary waves based on the compression forces on the particle contacts $\left(F_{1}, F_{2}\right)$. Both of these plots are shown in Fig. 2(b). In our calculations we also used the Hertz law for PTFE particle interaction. We used different values of elastic modulus and Poisson ratio equal to $0.46[34,40]$. The interaction between the flat wall and last particle was also described by the Hertz law with an elastic modulus of $115 \mathrm{GPa}$ and Poisson ratio of 0.307 for red brass $\left(\mathrm{Cu}_{85} \mathrm{Zn}_{15}\right)$. The Hertz law was also used to calculate the interaction between the alumina impactor $(E=416 \mathrm{GPa}, \nu=0.231)$ and the first PTFE particle. No dissipation was included in the numerical analysis. The gravitational force acting on particles, causing initial nonuniform precompression in the chain increasing toward the wall, was included in the calculations. The linear momentum (before interaction with a wall) and energy were conserved with a relative error of $10^{-12} \%$ and $10^{-8} \%$. Results of the numerical calculations modeling our experimental setup and conditions are shown in Figs. 2(b), 2(c), and 3; see the corresponding curves in Figs. 4(b), 5(b), 6(b), and 6(d).

\section{DISCUSSION}

PTFE is a polymeric viscoelastic material with a strong strain rate sensitivity [41] and exceptionally low elastic modulus [40]. At normal conditions the Young's and flexural moduli for PTFE are in the range of 400-750 MPa and the Poisson ratio is $0.46[34,40,42]$. This property can be very attractive for ensuring a very low speed of soliton propagation and tunability of the system. But it is not evident that a chain formed from this type of beads will support strongly nonlinear solitary waves as do chains made from typical linear elastic materials like stainless steel $[2,10,14]$. In particular, the role of dissipation and deviation from a linear elastic law [41] for PTFE under high strain and high strain rate deformation in the contact area with high gradients of strain is the primary concern. In the present study, the strains obtained were up to 0.06 , based on estimation of maximum compressive stresses in the center of the contact on the order of $80 \mathrm{MPa}$ at a maximum force approximately equal to $5 \mathrm{~N}$. The typical strain rates were approximately $4 \times 10^{2} \mathrm{~s}^{-1}$ and the compressive strains at the center of contact were about 0.06 decreasing to zero at a distance about $170 \mu \mathrm{m}$.

Beads made from nylon with elastic modulus six times larger than that for PTFE demonstrated a Hertz type interaction law [14]. Chains made of these beads supported propagation of strongly nonlinear solitary waves with amplitudes in the range 1-33 N. In our experiments we extended the range of amplitudes of solitary waves toward far smaller amplitudes by more than an order of magnitude, down to $0.03 \mathrm{~N}$. The dynamic behavior of sonic vacuum type systems at such low amplitudes is very interesting especially in view of potential practical applications related to noise reduction in the audible acoustic range, acoustic lenses, and delay lines, and for investigation of the validity of the Hertz law at very low displacements.

Furthermore, one of the distinguishing features of sonic vacuum systems is a strongly nonlinear dependence of the solitary wave speed on amplitude and precompression [Eqs. (3) and (4)], which are the important factors in imparting tunability of various properties of these systems, for example, in delay lines or acoustical lenses. The dependence of the solitary wave speed on the force amplitude $\left(F_{m}\right)$, based on Eqs. (4) and (3), is shown in Figs. 3(a) and 3(b) (curves 3 and 4 , respectively) with an elastic modulus equal to $600 \mathrm{MPa}$ [43]. Results of the numerical calculations for a discrete chain are also shown in Fig. 3(a) at the same value of elastic modulus (see curves 7 and 8). It is clear that theoretical and numerical approaches result in very close values of the speeds in the given interval of amplitudes. A noticeable deviation of the experimental data from curve 1 in Fig. 3(b) was observed for low amplitude solitary waves. Comparison of the solitary wave profiles and speeds in a discrete chain and in a continuum approximation for different nonlinear interaction laws is considered in $[17,29]$. 

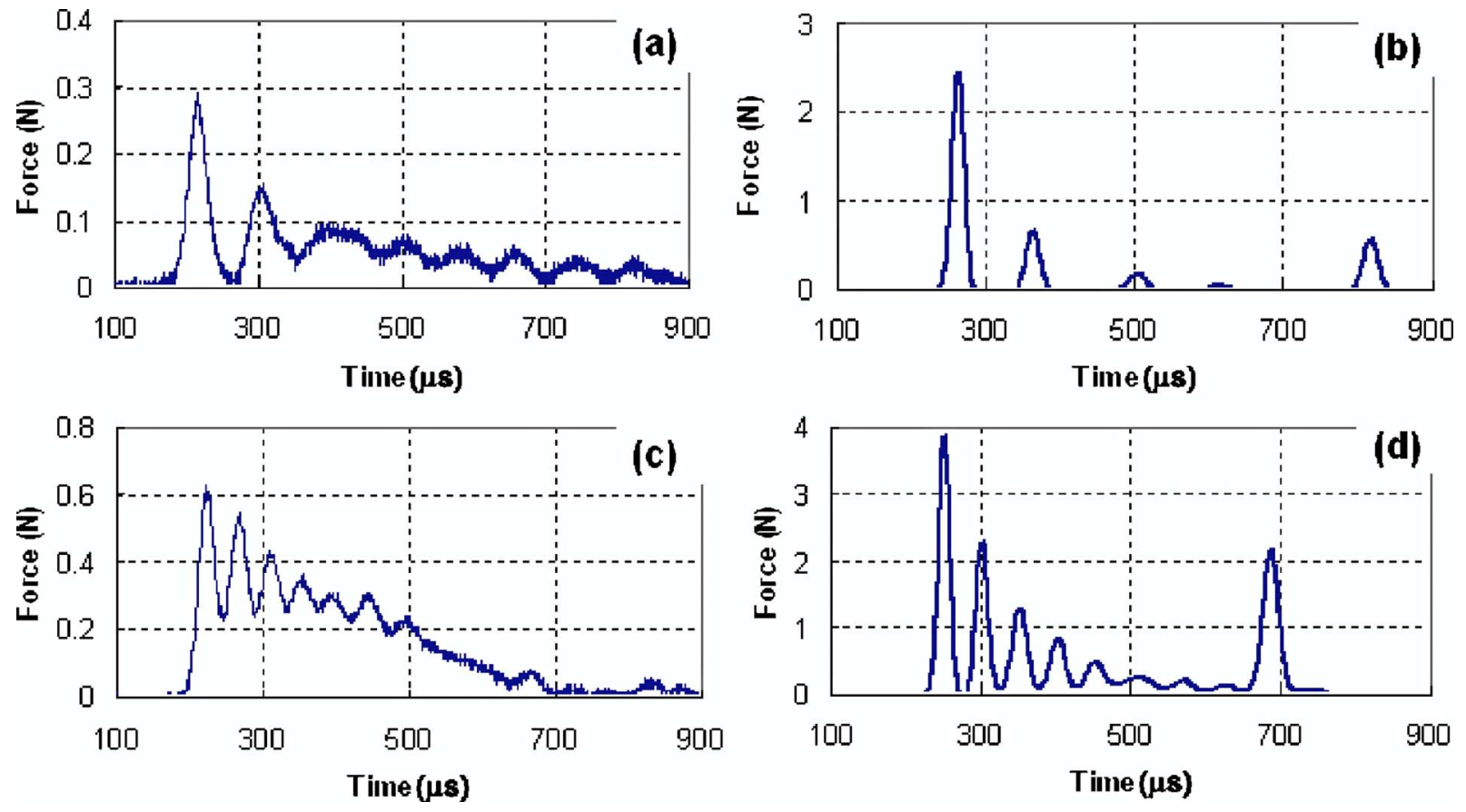

FIG. 6. (Color online) The experimental and theoretical results demonstrating that a PTFE chain of smaller particles with 2.38 mm diameter supports solitary waves and oscillatory "shock" waves modified by dissipation in experiments. (a) The leading solitary wave with an oscillatory tail detected at the wall generated in a chain of 18 smaller PTFE balls $(0.016 \mathrm{~g})$ [the velocity of the $2 \mathrm{~mm}$ diameter steel ball impactor $(0.036 \mathrm{~g})$ was $0.89 \mathrm{~m} / \mathrm{s}$ ]. (b) Numerical calculations corresponding to experimental conditions in (a), including gravitational precompression; elastic modulus $1.46 \mathrm{GPa}$. (c) Oscillatory "shock" wave detected at the wall generated in a chain of 18 PTFE balls, impacted at velocity $0.89 \mathrm{~m} / \mathrm{s}$ with a $5 \mathrm{~mm}$ diameter PTFE ball $(0.123 \mathrm{~g})$. (d) Train of solitary waves detected in numerical calculations corresponding to experimental conditions in (c), including gravitational precompression; elastic modulus $1.46 \mathrm{GPa}$.

It is evident that there is a large difference between experimental values of the soliton speed obtained in numerical calculations and in the long wave theory for large amplitudes of force if the value of the Young's modulus is taken as $600 \mathrm{MPa}$ [43]. If $400 \mathrm{MPa}$ was used for the PTFE elastic modulus [42], the difference between experimental speeds at high amplitude and predicted values based on the long wave approximation (or on numerical calculations) would be even more dramatic.

It should be mentioned that the dependence of shock wave speed $u_{s}$ on particle velocity $u_{p}$ in polymers [Hugoniotcurves in $u_{s}\left(u_{p}\right)$ coordinates] extrapolated to bulk sound speed results in significantly higher values than the sound speed at normal conditions measured using ultrasonic technique. This well known discrepancy indicates a rapidly varying change of compressibility at low values of shock amplitudes [44]. For PTFE, the extrapolated value of bulk speed $c_{b}$ from a Hugoniot curve gives a value of $1.68 \mathrm{~km} / \mathrm{s}$ in comparison with $1.139 \mathrm{~km} / \mathrm{s}$ from ultrasonic measurements. Using $c_{b}=1.68 \mathrm{~km} / \mathrm{s}$ from extrapolated Hugoniot measurements and Poisson ratio 0.46, we obtained a value of Young's modulus equal to $1.46 \mathrm{GPa}$ based on relations for elastic solids [45]. Ultrasonic data for the same material give a value of the elastic modulus equal to $704 \mathrm{MPa}$ [45]. The calculated theoretical and numerical data for solitary wave speed versus amplitude using an elastic modulus of $1.46 \mathrm{GPa}$ are presented in Fig. 3(a) (see curves 1, 2, 5, and 6). When comparing the analytical data [curves 1 and 2 in Figs. 3(a) and 3(b) at $E=1.46 \mathrm{GPa}$ ] with the experimental data [in Fig. 3(b)] we can see that there is a better agreement between the experimental results and the calculated speed of solitary waves at high amplitude.

Calculation with an elastic modulus of $1000 \mathrm{MPa}$ results in a reasonable correspondence between experimental data and calculation in the lower range of investigated force amplitudes of solitary waves. This suggests that the elastic modulus of PTFE is likely to be stress and strain rate dependent.

In a sonic vacuum, the solitary wavelength does not depend on amplitude; it depends on the behavior of the interaction force $[17,18]$. In the case of a power law Hertz interaction $[n=3 / 2$, Eq. (6)] this length is equal to five particles. The properties of solitary waves were used to establish the validity of the Hertz law for different materials [14]. Measuring solitary wave speeds and durations in our experiments allows straightforward calculation of solitary wave widths corresponding to different amplitudes (see Table I).

In the experiment corresponding to Fig. 4(a), for example, the speed of the leading solitary wave (with amplitude about $2 \mathrm{~N}$ ) was measured using the fifth and ninth particles from the wall and was found to be $152 \mathrm{~m} / \mathrm{s}$. The estimation based on the peak-to-peak measurements between the sensor in the fifth particle and the wall gave a similar value. The duration of solitary wave was about $164 \mu$ s resulting in a length of solitary wave equal to 5.2 times the diameter of the PTFE particle (Table I). This is very close to the predicted length of the solitary wave in the long wave approximation, equal to five particle diameters $[1,4,18]$. The measurement of aver- 
aged force using sensors embedded into the particle results in a slightly longer pulse in comparison with pulse duration based on the contact forces [Fig. 2(b)].

It is clear from the experimental data that the widths of solitary waves with relatively large amplitude are close to the predicted value of five particle diameters based on the Hertz interaction law. Numerical analysis of a solitary wave in a sonic vacuum demonstrated that the energy contained in five particles is equal to $99.999996 \%$ of the total energy of the solitary wave. The distribution of velocities of particles in a solitary wave including more than five particles was analyzed in $[16,46]$. It should be mentioned that these widths do not depend on the elastic modulus. The widths of the solitary waves tend to be wider at lower amplitudes of the propagating signals (Table I). This may be due to the influence of gravitational precompression closer to the impulse amplitude, which drives the system away from the strongly nonlinear limit, producing broader solitary pulses tending to the weakly nonlinear regime described by solitary waves of Korteweg-de Vries equation [4,18,25]. Numerical calculations performed in this work demonstrated a similar dependence of solitary widths on the amplitudes seen in Table I.

It is evident from the comparison between Fig. 4(a) and Fig. 4(b) that numerical calculations of the behavior of a discrete system and experimental results are in close agreement with respect to the signal amplitudes and time durations between corresponding pulses. It is noted, however, that the amplitude of the reflected solitary wave recorded by the sensor inside the ninth bead is significantly smaller than the amplitude of the incident wave. This is apparently due to the presence of dissipation in experiments, which was not taken into account in numerical calculations and will be addressed in future research.

From the preceding discussions it is apparent that a chain of low modulus PTFE beads also supports the propagation of a strongly nonlinear solitary wave, which is yet another realization of the sonic vacuum type of phononic crystal with exceptionally low speed of the signal.

Another remarkable feature of a sonic vacuum type system is the very fast decomposition of the longer initial pulse into a train of solitary waves $[1,2,4,17,18]$. Apparently this phenomenon can be obscured by the strong dissipation in the system. To check if this property is also demonstrated by a chain of PTFE particles, we used a striker mass $\left(m_{s}\right.$ $=0.47 \mathrm{~g}$ ) that was higher than the mass of the particles in the chain to create a longer incident pulse. Usually the number of solitary waves with significant amplitude is comparable to the ratio of the striker mass to the mass of the beads in the chain $[1,4,17,18,28]$.

The results of this experiment are shown in Fig. 5. It is evident that this Teflon-based sonic vacuum also demonstrates very fast decomposition of the initial impulse on a distance comparable with the soliton width [Figs. 5(a) and $5(b)]$ and a clear tendency of signal splitting is very noticeable already after only ten particles. The mass of the striker was chosen to be about four times that of the particles in the chain, expecting a decomposition of the initial triangular pulse into a train of four solitary waves. It should be mentioned that the number of solitary waves may be significantly larger if smaller amplitudes are included $[17,46]$. This ex- ample also demonstrates that a short duration impact on highly nonlinear sonic vacuum type ordered periodic systems results in a train of solitary waves instead of the intuitively expected shock wave. An increase of the duration of impact results in a shock wave impulse with an oscillatory structure where the leading pulse can be a KdV type solitary wave for a weakly nonlinear chain or a strongly nonlinear soliton with finite width for the strongly nonlinear case $[1,4,18]$. Similar qualitative agreement of the experimental results and numerical calculations was found for all investigated conditions of impact.

Previous experimental work $[2,7,8,10,14]$ with chains of steel beads, acrylic disks and spheres, and glass, brass, and nylon beads validated the prediction of a strongly nonlinear solitary wave as a stationary solution of the strongly nonlinear wave equation [Eq. (1)]. In those cases, the amplitude of the maximum force in the solitary wave was at least thirty times greater ( $1 \mathrm{~N}$ for nylon beads) than the one obtained in this paper for PTFE beads $(0.03 \mathrm{~N})$. This and the higher elastic modulus of nylon resulted in higher speeds of signal propagation (the minimum reported speed was $235 \mathrm{~m} / \mathrm{s}$ for nylon beads [14]). Furthermore, PTFE is a very versatile viscoelastic material. It is widely biocompatible and has a very low friction coefficient and a very low elastic modulus, which ensures applicability in a large variety of engineering solutions. As a result, we were able to experimentally achieve a speed of signal propagation of $88 \mathrm{~m} / \mathrm{s}$ for a force amplitude of $0.06 \mathrm{~N}$ (Table I), which is more than two times smaller than the speed of solitary wave detected for nylon beads [14] and more than three times smaller than the sound speed in the air at normal conditions. In principle, sonic vacuum type media of different structures (Hertzian and nonHertzian) can support solitary waves with indefinitely small amplitude and speed of propagation. In the future, it is not unreasonable to expect that a sonic vacuum type system which supports detectable solitary waves with force amplitude similar to the one investigated in this paper with a speed of the order of magnitude of $10 \mathrm{~m} / \mathrm{s}$ or lower could be designed using materials with tailored elastic properties.

Finally, it is important to investigate the influence of particle size on the system behavior for application purposes. In fact, smaller size of the particles composing the PTFE-based strongly nonlinear system results in different stresses and strain rate conditions in the contact area which may affect the system behavior. We conducted experiments with smaller diameter PTFE balls $(2.38 \mathrm{~mm})$ to check the validity of the strongly nonlinear theory. Experimental and numerical results are presented in Fig. 6. In the experiments, pulses were generated by impact of a $2 \mathrm{~mm}$ diameter steel ball $[0.036 \mathrm{~g}$, Figs. 6(a) and 6(b)] and a $5 \mathrm{~mm}$ diameter PTFE ball $[0.123 \mathrm{~g}$, Figs. 6(c) and 6(d)] at velocity $0.89 \mathrm{~m} / \mathrm{s}$. Numerical calculations did not account for the effects of dissipation. It is evident that the smaller diameter PTFE particles do support the sonic vacuum type behavior, although in this case the effect of dissipation appears to be more significant. The influence of dissipation on dynamics of solitary waves in strongly nonlinear discrete systems was considered in $[20,26]$. The effect of dissipation is likely to be responsible for the tail present after the second solitary waves formed in experiments [Fig. 6(a)], and delays the solitary wave split- 
ting in experiments in comparison with numerical results [compare Fig. 6(a) and 6(b) and Fig. 6(c) and 6(d)].

\section{CONCLUSIONS}

Propagation of impulses in one-dimensional strongly nonlinear phononic crystals assembled from PTFE spheres was investigated for different conditions of loading and geometrical parameters. It was demonstrated that the chains of PTFE beads with different diameters support the Hertzian behavior with very low signal propagation speed due to its exceptionally low Young's modulus and despite the viscoelastic nature of PTFE. Single solitary waves and decomposition of the signal into trains of solitary waves with amplitude more than one order of magnitude smaller than previously reported were observed. The small amplitude solitons broke the "sound barrier," having a speed of propagation well below sound speed in air. Single solitary waves and trains of strongly nonlinear solitary waves excited by impact were investigated experimentally and were found to be in reasonable agreement with numerical calculations based on the Hertz interaction law with Young's modulus $1000 \mathrm{MPa}$ for lower amplitudes and $1460 \mathrm{MPa}$ for higher amplitudes of signals, both being significantly higher than its value in normal conditions.

\section{ACKNOWLEDGMENT}

This work was supported by NSF (Grant No. DCMS03013220).
[1] V. F. Nesterenko, Prikl. Mekh. Tekh. Fiz. 5, 136 (1983) [J. Appl. Mech. Tech. Phys. 5, 733 (1984)].

[2] A. N. Lazaridi and V. F. Nesterenko, Prikl. Mekh. Tekh. Fiz. 3, 115 (1985) [J. Appl. Mech. Tech. Phys. 3, 405 (1985)].

[3] V. F. Nesterenko, Fiz. Goreniya Vzryva 28, 121 (1992).

[4] V. F. Nesterenko, High-Rate Deformation of Heterogeneous Materials (Novosibirsk, Nauka, 1992), Chap. 2 (in Russian).

[5] V. F. Nesterenko, Fiz. Goreniya Vzryva 29, 132 (1993).

[6] S. L. Gavrilyuk and V. F. Nesterenko, Prikl. Mekh. Tekh. Fiz. 6, 45 (1993) [J. Appl. Mech. Tech. Phys. 6, 784 (1993)].

[7] A. Shukla, M. H. Sadd, Y. Xu, and Q. M. Tai, J. Mech. Phys. Solids 41, 1795 (1993).

[8] Y. Zhu, F. Sienkiewicz, A. Shukla, and M. Sadd, J. Eng. Mech. 10, 1050 (1997).

[9] V. F. Nesterenko, J. Phys. IV 4, C8-729 (1994).

[10] C. Coste, E. Falcon, and S. Fauve, Phys. Rev. E 56, 6104 (1997).

[11] M. Manciu, S. Sen, and A. J. Hurd, Physica A 274, 607 (1999).

[12] M. Remoissenet, Waves Called Solitons (Concepts and Experiments), 3rd revised and enlarged ed. (Springer-Verlag, Berlin, 1999).

[13] E. J. Hinch and S. Saint-Jean, Proc. R. Soc. London, Ser. A 455, 3201 (1999).

[14] C. Coste and B. Gilles, Eur. Phys. J. B 7, 155 (1999).

[15] J. Hong, J.-Y. Ji, and H. Kim, Phys. Rev. Lett. 82, 3058 (1999).

[16] A. Chatterjee, Phys. Rev. E 59, 5912 (1999).

[17] E. Hascoet and H. J. Herrmann, Eur. Phys. J. B 14, 183 (2000).

[18] V. F. Nesterenko, Dynamics of Heterogeneous Materials (Springer-Verlag, New York, 2001), Chap. 1.

[19] S. Sen, M. Manciu, R. S. Sinkovits, and A. J. Hurd, Granular Matter 3, 33 (2001).

[20] M. Manciu, S. Sen, and A. J. Hurd, Physica D 157, 226 (2001).

[21] M. Manciu, S. Sen, and A. J. Hurd, Phys. Rev. E 63, 016614 (2001).

[22] J. B. Hong and A. G. Xu, Phys. Rev. E 63, 061310 (2001).

[23] S. Sen and M. Manciu, Phys. Rev. E 64, 056605 (2001).

[24] F. S. Manciu and S. Sen, Phys. Rev. E 66, 016616 (2002).

[25] C. A. Arancibia-Bulnes and J. C. Ruiz-Suarez, Physica D 168, 159 (2002).
[26] A. Rosas and K. Lindenberg, Phys. Rev. E 68, 041304 (2003).

[27] R. Reigada, A. Samiento, and K. Lindenberg, Chaos 13, 646 (2003).

[28] C. Daraio, V. F. Nesterenko, and S. Jin, in Shock Compression of Condensed Matter-2003, edited by M. D. Furnish, Y. M. Gupta, and J. W. Forbes, AIP Conf. Proc. No. 706 (AIP, Melville, NY, 2004), pp. 197-200.

[29] A. Rosas and K. Lindenberg, Phys. Rev. E 69, 037601 (2004).

[30] A. I. Musienko and L. I. Manevich, Phys. Usp. 47, 797 (2004).

[31] M. Kastner, Phys. Rev. Lett. 92, 104301 (2004).

[32] M. Kastner, Nonlinearity 17, 1923 (2004).

[33] E. Fermi, J. R. Pasta, and S. M. Ulam, Los Alamos National Laboratory Technical Report No. LA-1940, reprinted in Collected Works of E. Fermi (University of Chicago Press, Chicago, 1965), Vol. 2, pp. 978-988.

[34] DuPont product information, www.dupont.com/teflon/ chemical/

[35] C. Daraio, V. F. Nesterenko, and S. Jin, Appl. Phys. Lett. 85, 5724 (2004).

[36] V. F. Nesterenko, A. N. Lazaridi, and E. B. Sibiryakov, Prikl. Mekh. Tekh. Fiz. 2, 19 (1995) [J. Appl. Mech. Tech. Phys. 36, 166 (1995)].

[37] S. Job, F. Melo, A. Sokolow, and S. Sen Phys. Rev. Lett. 94, 178002 (2005).

[38] H. Hertz, J. Reine Angew. Math. 92, 156 (1881).

[39] S. P. Timoshenko and J. N. Goodier, Theory of Elasticity, 3rd ed. (McGraw-Hill, New York, 1987), p. 414.

[40] G. W. Ehrenstein, Polymeric Materials: Structure, Properties, Applications (Hanser, Munich/Hanser Gardner Publications, Cincinnati, OH, 2001), p. 256.

[41] F. J. Zerilli and R. W. Armstrong, in Shock Compression of Condensed Matter-2001, edited by M. D. Furnish, N. N. Thadhani, and Y. Horie, AIP Conf. Proc. No. 620, (AIP, Melville, NY, 2002), pp. 657-660.

[42] P. Gondret, M. Lance, and L. Petit, Phys. Fluids 14, 643 (2002).

[43] AcraBall Manufactoring Co. Product catalog.

[44] W. J. Carter and S. P. Marsh, Los Alamos National Laboratory Report No. LA-13006-MS, 1995 (unpublished).

[45] Y. C. Fung, Foundations of Solid Mechanics (Prentice-Hall, Englewood Cliffs, NJ, 1965).

[46] A. Sokolow, E. G. Bittle, and S. Sen (unpublished). 